Blucher

\author{
Blucher Proceedings \\ Cuba e Brasil no Século XXI (CBS21) \\ A Construção do Conhecimento
}

\title{
Financiamiento de las Investigaciones y Políticas Nacionales de Desarrollo
}

\author{
Dr. Carlos Trallero-Giner ${ }^{1}$
}

\section{Introducción}

Sirvan estas reflexiones sobre las políticas científicas y en particular destacar el rol que en esta dirección ocupa el Centro Latinoamericano de Física (CLAF), su historia, su presente y sus perspectivas, y quizá si no fuera muy pretencioso de mi parte una invitación a la complicidad después de sus 50 años de existencia.

Para tener una idea de cómo se han ido tejiendo en los años las políticas sobre el desarrollo científico en nuestros países, se hace necesario tener una visión del marco histórico en el cual se produce el surgimiento del CLAF.

\section{Recorrido a través de la Historia del CLAF}

Los resultados exitosos de los lanzamientos de sputniks desde el 1957, por la entonces Unión de Repúblicas Socialistas Soviéticas, hasta agosto de 1960 transportando al cosmos instrumentos de investigación sobre geofísica, animales y plantas, conmovió profundamente a la comunidad científica internacional. Junto a estos resultados se añaden la presencia del primer cosmonauta el 12 de abril de 1961 y la carrera armamentista que produjo la bomba nuclear más poderosa (Tsar Bomba o Bomba Emperador) que haya existido en la historia de la humanidad, lanzada el 30 de octubre de 1961.

\footnotetext{
${ }^{1}$ Diretor do Centro Latino-Americano de Física (CLAF), Rio de Janeiro, Brasil. E-mail: trallerocarlos@gmail.com.
} 
Esta confrontación política, ideológica y económica entre las mayores potencias del planeta condujo a un incremento de inversiones para la educación e investigación científica. Un ejemplo notable de ello fue el apoyo brindado en los Estados Unidos al sector educacional. Durante el año 1956 en el Massachusetts Institute of Technologic (MIT) se creó el programa denominado "Physical Science Study Committee" (PSSC) dedicado a mejorar la enseñanza introductoria de los cursos de física. El comité creado inicialmente consideraba que la enseñanza de la ciencia en EUA era deficitaria y se argumentaba que el avance de la ciencia estaba comprometido. El gobierno de los EUA inicialmente otorgó a este proyecto la cantidad de 5 millones de dólares USD. Para la época esta era una suma inmensa de dinero destinada a proyectos docentes. No obstante, luego de los acontecimientos del sputnik y demás, el PSSC recibió un monto de 200 millones de dólares USD lo cual demuestra la preocupación existente de la época en cuanto al desarrollo de las ciencias básicas y su papel en la sociedad.

En este mismo período se crearon diferentes instituciones de física en diversas partes del mundo. En este contexto de la guerra fría, los gobiernos se sentían compulsados a realizar y financiar políticas científicas para apoyar las ciencias básicas. Así las cosas, en la época se crearon programas de postgrado en varios países de América Latina. En Brasil, por ejemplo, el programa de postgrado fue creado en 1961 en lo que hoy se conoce como Centro Brasileiro de Pesquisas Físicas $(\mathrm{CBPF})$. En Cuba en la misma fecha se inicio la Escuela de Física en la Universidad de La Habana, entre otros.

El Centro Latinoamericano de Física con sede en el CBPF en Rio de Janeiro inició sus labores hace 51 años con el consenso de la mayoría de los países de la región.

Se debe destacar que precursoras del CLAF fueron las Escuelas Latinoamericanas realizadas entre 1959 y 1961 las que reunieron a relevantes personalidades en el campo de la física y tuvieron gran influencia en el desarrollo de investigaciones en diversos países de América Latina.

Estimulados por el éxito de estas escuelas y bajo los auspicios de la UNESCO, los físicos latinoamericanos Juan José Giambiagi (Argentina), José Leite Lopes (Brasil) y Marcos Moshinky (México), elaboraron el proyecto de creación del Centro Latinoamericano de Física, que fue fundado el 26 de marzo de 1962, siendo su primer Director Gabriel E.A. Fialho.

En un inicio el CLAF priorizó la formación académica gracias a un agresivo programa de becas. Se encaminaron los recursos disponibles para la obtención de grado científico lo que favoreció a países menos desarrollados. Al finalizar los 60 y en los años 70 cuando ya se había establecido la capacidad de formación propia en los diversos países miembros del CLAF, se apoyó el intercambio de físicos, la creación de Escuelas, Grupos de Trabajo y Conferencias Latinoamericanas. Cabe 
señalar la realización de la Escuela Latinoamericana de Física en Oaxtepec, México en 1968, marco verdadero para la física latinoamericana dada la expresiva participación de la mayoría de los físicos de la región. Un papel líder para toda América Latina lo jugó Brasil graduando una gran cantidad de doctores de diversos países.

En la década de los años 80 la atención del CLAF se volcó también hacia actividades interdisciplinares de interés regional con la perspectiva de encontrar mayor integración entre la física y los físicos con otras aéreas afines de la ciencia. Se destaca el impulso a eventos científicos latinoamericanos como son los Simposios Latinoamericanos de Física del Estado Solido, (SLAFES), y Congresos Latinoamericanos de Ciencias de Superficie y sus Aplicaciones, (CLACSA). Siguiendo esta dirección se buscó ampliar los horizontes de trabajo para físicos y paralelamente demostrar a la sociedad la importancia de la ciencia y la tecnología para el desarrollo de Latinoamérica, a través de la solución de problemas específicos de impacto económico y social. Al mismo tiempo se estimuló un programa de becas de post graduación como una forma de favorecer la colaboración e incrementar la investigación entre los distintos países del área.

Con posterioridad a los años 90 se intenta encontrar recursos para promover programas a largo plazo tendentes a la mejor utilización de instalaciones experimentales existentes o proyectadas en América Latina.
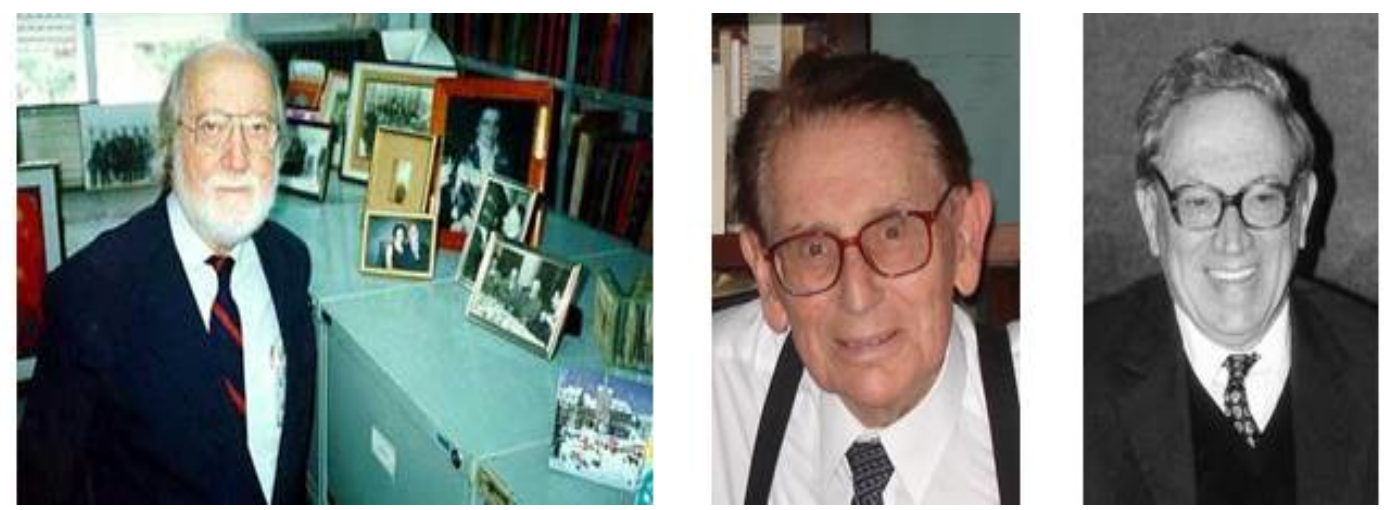

Figura 1: De izquierda a derecha, respectivamente, las fotos de José Leite Lopes, Marcos Moshinsky y Juan José Giambiagi.

\section{EI CLAF. Una Mirada Actual}

Hoy en día se trabaja en ampliar los horizontes para una mayor participación en diversos proyectos como son el "Brazilian Synchrotron Light Laboratory" (LNLS), el "Agua Negra Deep Experiment Site"(ANDES), la utilización de red internacional GRID, mediante convenio, CERN-CLAF, entre otros. 
Es una realidad que en la actualidad no siempre la ciencia en Latinoamérica es aceptada como en los 60, vale como anécdota lo que comento Guido Beck (físico austriaco vinculado a diversos grupos latinoamericanos). Normalmente, los gobiernos dicen: "Nosotros no necesitamos de ciencia; nosotros precisamos de aplicaciones". Esto es lo mismo que afirmar: "Yo preciso de la leche, la vaca no me interesa." Pero, sin vaca, no hay leche. Ellos tienen que comprender que precisan de la vaca y de la leche al mismo tiempo. Para tener leche es preciso alimentar la vaca. Eso toma un cierto tiempo. ${ }^{2}$

Después de 50 años y atendiendo al contexto actual, tanto de Latinoamérica como mundial, es necesario profundizar en los objetivos, perspectivas y alcance, que debemos tener presente para lograr un financiamiento adecuado y políticas de desarrollo responsables hacia la región. En la actualidad, la presencia de diferencias económicas así como de los más diversos problemas que atañen a cada área geográfica nos encontramos frente a un desarrollo desigual en Latinoamérica, por lo que se hace necesario un cambio de paradigmas.

En los años 60-70, de importancia vital era la formación de personal, hoy, sin restarle importancia debemos racionalizar nuestros recursos, encontrar mecanismos para una mejor utilización y explotación de los medios asignados, aumentar la integración científica entre los colectivos científicos de la región, entre otros.

Para comprender mejor nuestra realidad vale entender que en los 60-70 la diferencia científica entre los países integrantes de Latinoamérica no era muy diferente. Hoy en día, hay una desigualdad notable de desarrollo entre diversas áreas del continente e incluso en un mismo país. Esto determina que el lenguaje a seguir e ideas a debatir deban ser articulados de una forma distinta con cada grupo y región. La búsqueda de sectores con condiciones de desarrollo similar es también una prioridad, la comunicación y estudio de problemas comunes permite un mayor desarrollo de la ciencia, articulada a los problemas propios de estas comunidades.

El avance acelerado en los últimos años de la Física en Brasil hace que el CLAF tome como referente a diversas instituciones de este país y procure vínculos de intercambio entre estas y otros grupos de trabajo nacionales e internacionales.

Una manera muy prioritaria de conseguir recursos es mediante la explotación más racional de la infraestructura ya instalada o en proyección. Pongamos por ejemplo el número de proyectos reportados en el 2010 en el LNLS: Brasil desarrolló 432, Argentina 74 Cuba 8, Chile 2 y Colombia 2. Es sin lugar a dudas que el sincrotrón situado en Campinas, Sao Paulo, es uno de los mejores recursos que contamos hoy día. La utilización más adecuada de este tipo de laboratorio nos dará un salto cualitativo en nuestra investigación, al igual que demostrar el impacto que debe tener en nuestras sociedades.

En la actualidad se hace necesario el análisis diferenciado de los países y regio-

\footnotetext{
${ }^{2}$ Tomado del trabajo sobre G. Beck de A. Augusto Passos/CBPF - cbpfindex.cbpf.br.
} 
nes buscando alternativas útiles para todos los miembros del CLAF. Es claro que en el futuro se debe seguir trabajando en la formación y mejoría de los recursos humanos y aumentar el intercambio entre diferentes organismos o instituciones de los países miembros. De importancia seguirá siendo el apoyo a escuelas, cursos y eventos nacionales e internacionales desarrollados en Latinoamérica. Debemos destacar que al margen de los recursos conseguidos para becas de doctorado y postdoctorales como son los convenios firmados con el CNPq de Brasil, el CONACYT de México, el Centro Internacional de Física Teórica (ICTP) de Trieste, Italia, se destaca la búsqueda de recursos financieros que permitan direccionar estancias de doctorado y postdoctorales a fines precisos de mayor necesidad en el desarrollo de la física. Así, temáticas como Sistemas Complejos e Historia de la Física en América Latina se vienen trabajando con lo Max Planck Institute for Complex Systems (MPIPKS) e History of Sciencies (MPIWP), respectivamente lo cual permitirá núcleos de desarrollo en importantes áreas del quehacer científico y técnico.

Otra línea no menos importante se vincula al papel que el CLAF debe jugar como eslabón regional en la colaboración científica en América Latina y con el resto de la comunidad científica internacional. Como un ejemplo positivo tenemos la firma del acuerdo recientemente firmado con el CERN que ha permitido a las universidades e instituciones científicas de América Latina participar en el proyecto "World wide LHC Compating Grid". Estamos enfrascados en un convenio similar con Europa, el conocido "European Grid Initiative Foundation" que permitirá participar en una red mundial de cómputo para cumplimentar trabajos en áreas de la biofísica, medio ambiente, sistemas complejos, química-física, entre otras.

Dado el desarrollo económico diferenciado de los países de América Latina se hace imprescindible establecer un intercambio más dinámico entre los grupos de investigación afines. Identificar grupos líderes en cada región y obtener un catálogo de recursos humanos e infraestructura son pasos iniciales y necesarios para lograr un financiamiento sustentable. Con muy pocos recursos los diversos grupos de la región (tanto de física como muchas otras áreas afines), pueden hacer uso de técnicas avanzadas de medición e insertarse en el quehacer mundial y de sus propios pueblos. El CLAF está dispuesto a coordinar y apoyar iniciativas en esta dirección.

La particular colaboración entre Cuba y Brasil es un digno ejemplo en esta dirección. Data de varios años atrás que investigadores de ambos países tienen conocimientos de los diferentes grupos de trabajo, equipamientos y laboratorios que están disponibles. Con este conocimiento de ambas partes ha sido posible argumentar el número de proyectos de investigación, estancias de trabajo, becas de doctorado y posiciones de postdoctorados. Son muy destacadas las actividades desarrolladas con el CNPq, CLAF, CAPES, FAPESP y Ministerios de Educación 
y de Ciencias de Cuba.

En la actualidad el CLAF se empeña aún más en desarrollar proyectos que apunten a la búsqueda de perfiles de investigación bien definidos que permitan una articulación adecuada entre los diversos grupos de trabajo. En ese sentido, el apoyo a eventos bien estructurados entre las diversas regiones afines en su quehacer nacional es una prioridad para el CLAF. Para la consecución de este objetivo se busca financiar estancias de trabajo conjunto, cursos de adiestramiento y postdoctorales dirigiendo los recursos a áreas del conocimiento prioritarias que darán mayor impulso a la actividad científico-técnica.

En este contexto y desde el punto de vista conceptual una tarea de primordial importancia es la investigación y divulgación de la Historia de la Ciencia Latinoamericana. El conocimiento de nuestros aportes científico, el recoger nuestras propias vivencias de acuerdo a nuestras características e idiosincrasias, nivel de desarrollo y necesidades, debe ser un objetivo permanente de las instituciones vinculadas al proceso educativo y científico.

Esperamos que estas contribuciones traigan consigo un mayor entendimiento entre nuestros pueblos, concientización de las estructuras gubernamentales y de la administración pública en general para alcanzar de forma acelerada valorizando nuestro medio ambiente y garantizando un desarrollo sustentable, el papel y lugar que nos corresponde en la ciencia mundial. 